\title{
STAGE IV BREAST CANCER SURGERY. CASE SERIES
}

María Liz Bareiro Paniagua

${ }^{1}$ Pontifícia Universidade Católica do Rio de Janeiro - Gávea (RJ), Brazil.

Introduction: Distant site tumor implantation implies a severe condition, stimulated by an additional genome evolution that compromises the survival (6 months in the visceral metastasis). The literature is still controversial, and the guidelines recommend surgery only as palliative care. New results are conflicting, however, the initial modern systemic therapy, followed by the resection of the primary tumor, seems to improve the global survival (GS). "De Novo Metastasis (MT)" is that found in the diagnostic investigation. Objective: To assess the results of the primary metastatic breast cancer (BC) surgery. Method: retrospective review of medical records in a cohort of patients with metastatic BC treated between 2011 and 2018 in the state of Rio de Janeiro. Result: Eleven patients were included. (Group A= 6 patients with De Novo $\mathrm{MT}$, and Group B= 5 patients with MT in the follow-up). In both cases, invasive ductal carcinoma was prevalent, frequently of grade 3 and with high Ki-67, Luminal B and Her-2. There was a mutation (BRCA2), and the PET CT was correlated to the complete pathological response. In group A, the patients were younger ( $50 \%<40$ years old), 5/6 initiated neoadjuvant chemotherapy (NACT), all underwent mastectomy (multicentricity or size), 33\% presented with imaging and pathological complete response (pCR). Of the MT, $50 \%$ were in the bone; $1 / 6$ had negative and operated liver and pulmonary MT (HER-2), with disease-free survival (DFS) superior to 12 months. In group B, all MTs came after local recurrence (LR) (between 3 and 11 months); 40\% had high initial axillary compromise (pN= mean of 25), and Luminal B, being treated according to the guidelines. In 2/5 (40\%) of visceral MT, 18 months of DFS were observed after pCR and primary tumor resection. Finally, in the follow-up (mean of 42 months), there were two casualties (TN) in group A, and 4/6 presented DFS between 17 and 72 months. In group B, 3/5 presented DFS between 12 and 24 months. Conclusion: In this sample, the pathological complete response suggested better prognosis before surgery. There was benefit in the survival of visceral MT (including multiple ones) with pCR and breast surgery in comparison to the literature; further studies are required, considering the limitations of this analysis. 